\title{
ONTOLOGIES IN COMPUTATIONAL ENGINEERING
}

\section{HEINZ A. PREISIG ${ }^{1}$, THOMAS F. HAGELIEN ${ }^{2}$, JESPER FRIIS ${ }^{2}$, PETER KLEIN ${ }^{3}$, NATALIA KONCHAKOVA ${ }^{4}$}

${ }^{1}$ Norwegian University of Science and Technology, Department of Chemical Engineering, NO-7491 Trondheim, Norway, heinz.a.preisig@ ntnu.no,

${ }^{2}$ SINTEF, NO-7465 Trondheim, Norway, Thomas.F.Hagelien@sintef.no, jesper.friis@ sintef.no

${ }^{3}$ Fraunhofer ITWM, Fraunhoferplatz 1, Kaiserslautern, 67663, Germany, peter.klein@itwm.fraunhofer.de

${ }^{4}$ Institute of Surface Technology, Helmholtz-Zentrum Geesthacht, Max-Planck Str. 1, Geesthacht, 21502, Germany, natalia.konchakova@hzg.de

Key words: Multiphysics simulations, Ontology-based framework, Digitalization strategy, Interoperability

\begin{abstract}
Industry and science define traditionally many needs for simulations. So one may ask what can be new in a field that is so well covered over so many years. It is not only the computing hardware that has undergone revolutionary developments. In parallel, software engineering kept pace - more and more high-level abstractions make their way into technology, making software systems increasingly powerful. The industry has an increasing need for multidisciplinary simulation, thereby generating demand for an extension towards integrating more different disciplines. Ontologies provide a perfect vehicle for the representation and coupling of knowledge, thus the integration. So the aim is to introduce ontologies on all levels into the larger domain of simulation software systems and construct a generic simulation ontology-based framework. The approach is focused on business-decisions and translation support using layers of simulation tools, including multiscale systems simulations of physics-based models. Latter describes the processing units' internals being coupled with control, optimisation and performance analysis based on ecology and techno-economical criteria.
\end{abstract}

\section{Introduction}

The used models and relations are mathematical descriptions of reality. The theoretical concepts of such frameworks are captured in ontologies, which is discipline-specific knowledge. The overarching concepts give answers to the questions: "What sort of ontology generates a specific model? ", "What are the possible ontological components capture the knowledge of a specified domain?", "What is the range of the application of a specific ontology?", "Why do specific domains need specific terminology and syntaxes?", "How can one construct the ontologies for a specific domain?". Finding the answer to these questions is the focus of this paper, aiming at the ultimate goal to design and operate processes systematically and generically. 
Ontologies have, by definition, an extensive range of applications. The materials community is in the process of generating marketplaces for process engineering activities related to materials and their processing. Besides materials behaviour descriptions, it extends to business decision support and product design. The range of potentially involved models thus stretches from business models to fundamental material models. It extends to the economy and ecology, all of which must be extracted from either simulated or experimental data. Moreover, ontology-based modelling supports the digitalisation strategy of material science and manufacturing for the European industry.

While we do not discuss the subject, optimisation enters the activity at different places - decision support, process and product design, operations, and solving physical models in time and space on different scales based on different business related key performance indicators (KPIs) [1]. Again, this activity is supported by ontologies both with regard to realising interoperability and problem definition.

Solving such broadly-defined engineering tasks requires the use of different programs, all of which interact and require access to data storage in the form of databases. Interoperability is again a problem that can be elegantly solved using the ontological description of data, nature, and structure.

While materials and optimising process modelling have been proven to deliver significant contributions in shortening the development time, business control over new materials, manufacturing processes, and products have not yet well taken up by industries. Besides, new technology is risky, time-consuming, and high-skilled demanding in many cases.

\section{Materials Modelling Market Place - Computation bazaar}

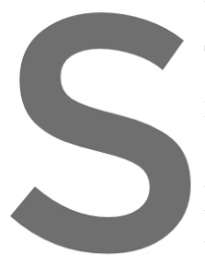

The European Commiss material-related computat and LEs $)^{1}$. Earlier project

Modelling Council ${ }^{2}$

tions tools covering the su
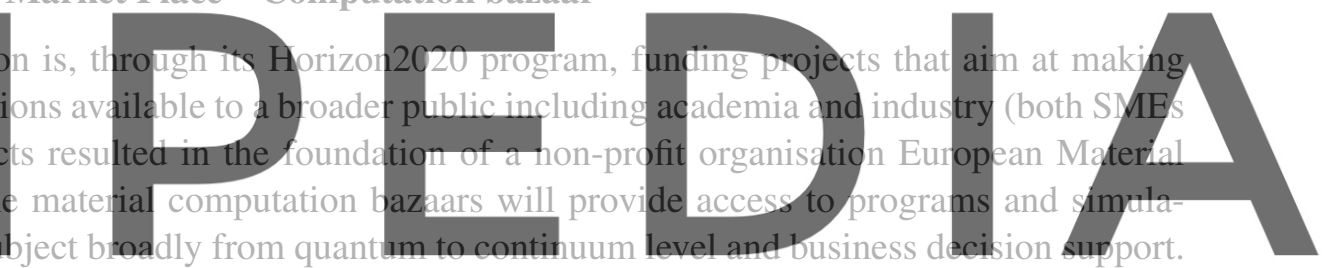

It shall thus cover the full range required to perform multi-scale, multi-physics and multi-disciplinary

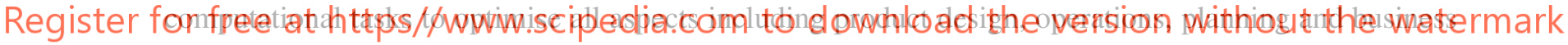
decision making. Not at least, the bazaar also gives access to consulting services and training's materials enabling closing the gap between the user and the experts.

\section{Why ontologies?}

Ontologies capture the structure and knowledge of a specific discipline. They are thus context-dependent. With material modelling being a multi-disciplinary subject and the bazaars having the objective to provide support across a wide range of disciplines, it is natural to utilise a combination of ontologies to meet the goals.

The ontologies serve the purpose of defining a terminology, a classification of relevant objects and rules on how to combine the various objects. The ontologies cover the field of business, the mathematical description of the physical constituents for the whole range of basic physical objects, and problem formulation and computational workflows.

\footnotetext{
${ }^{1} \mathrm{SME}$ - Small and Medium size Enterprises; LE - Large Enterprises

${ }^{2}$ emmc.eu/
} 
Since the computational tasks usually involve several programs, including database support, interoperability takes a central stage on the technical level. Also, this aspect is best handled by defining the data structures employing ontologies. It makes data structure definitions computer-readable and enables the automatic generation of the application interfaces (APIs) and their implementation through semantic services.

\subsection{How you can prepare the ontology for your simulations?}

The generation of a simulation requires first to collect the information that specifies the problem. The problem is recursively broken down to a level where one can identify a small set of basic objects with a known behaviour that is basic in the context of the discipline and exposes the relations between them. Both the objects and the identified relations that exist between them are then captured in an ontology.

\subsection{How are ontologies used}

Datamining is probably one of the main applications of ontologies, at least currently. Technically these are about recognising patterns. Applications may match corresponding parts in a problem definition or an existing workflow, match the user's need with a translator's background, existing process models, or parts of them, or suggested objectives with existing optimisation formulations.

However, they are also used in a variety of other domains. Rather than using them for pattern recognition, one can also use them as a "construction regulation". For example, for the definition of for businessrelated workflows. BPMN (Business Process Model and Notation) is a well-known technology and has

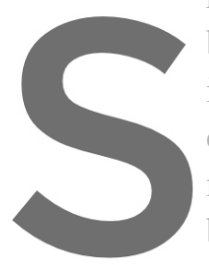
been captured in several ontologies tuned to specif
is currently working on generating an ontology fo
operational blocks, and NTNU's Process Systems
models, whereby the ontology provides the building
basic building blocks and their mathematical behav
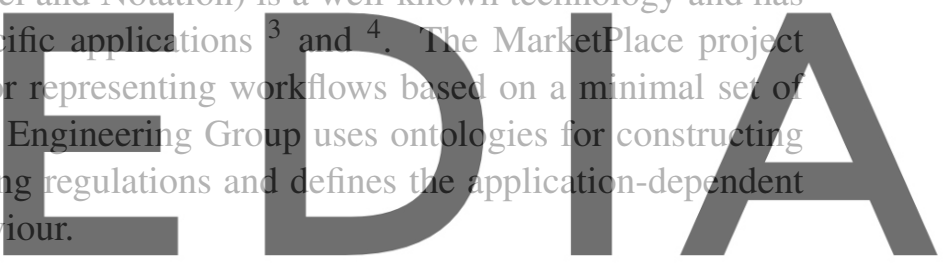

One may also use them for model construction and the automatic construction of application interfaces to

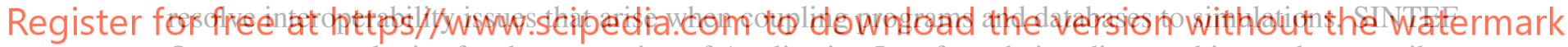
Ocean uses ontologies for the generation of Application Interfaces being discussed in another contribution to this conference [3].

Ontologies have another vital function: they classify items, thus define a taxonometry, and they define relations between them. In this light, the European Material Modelling Council (EMMC-ASBL) ${ }^{5}$ is in the process of defining a European Material Modelling Ontology (EMMO) [2], which has the objective to standardise the "material modelling language" for the integration of materials modelling and the digitalisation for product and process design.

\section{The Process Modelling Suite ProMo: Structure \& mission}

The Process Systems Engineering Group at NTNU is constructing an ontology-based simulation environment. The project has a long history, starting in the mid-1980s with modelling life support sys-

\footnotetext{
${ }^{3}$ Data \& Knowledge Management as part of Center for Information Technology - Fondazione Bruno Kessler

${ }^{4}$ BBO: BPMN 2.0 IRIT, CNRS, University of Toulouse, Toulouse, France

5 https://emmc.eu/
} 
tems for space applications. It has evolved gradually and was first commercialised by MobaTech in the Netherlands. The continuation expands into multi-disciplines and multi-scale primarily motivated by the Framework7 project MoDeNa [4]. The new project is known as ProMo. It has three major sections:

ProMo-Expert which defines the ontology for an application domain yielding a tree-like structure of the involved disciplines. Next, the disciplines' basic entities are equipped with mathematical equations representing their dynamic behaviour and linking them to graphical objects. The behaviour equations are compiled and made available of the code generation representing specific models by the translator. They are also translated into LaTex for documentation and OWL for the APIs' automatic generation realising interoperability.

ProMo-Translator constructs the model using the basic building blocks in a graphical editor. The result is a hierarchical topology coloured with the materials' components, which then is used to generate the executable workflow or simulation task.

\section{ProMo-User employs the simulation for the specified purpose.}

Figure 1 shows the main components of the ProMo simulation environment.
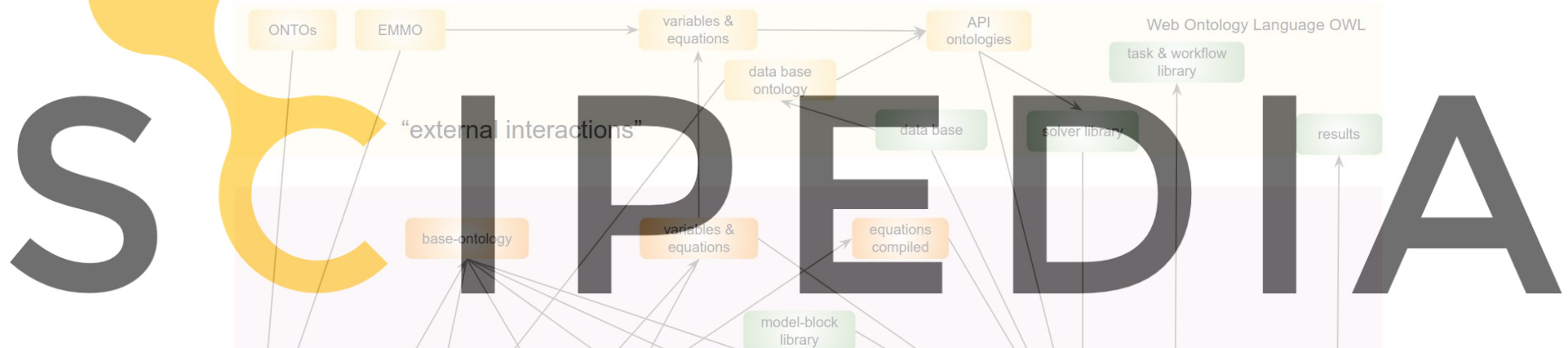

Register for free at https//www.scipedia.com to download the version without the watermark
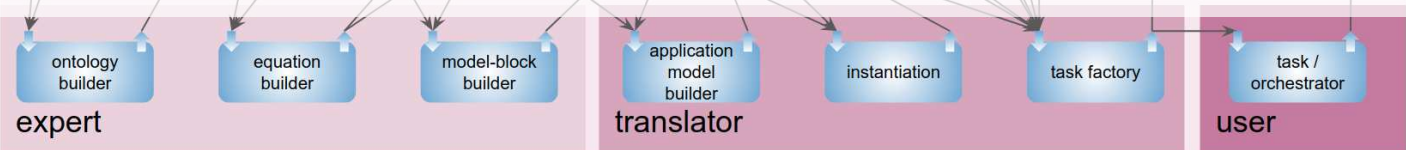

Figure 1: A view on the ProMo architecture. There are three major sections: the expert, the translator and the user section. As the name indicates, they are aiming at specified groups of people. The top part is dedicated to the ontologies, while in the middle section is reserved for the ProMo libraries and the lowest section contains the major executable ProMo tasks.

The top section shows the OWL (triple-store) ontology parts, with the main object being the variables $\&$ equations ${ }^{6}$ generated by ProMo. The middle section contains the ProMo libraries, and the lowest section captures the main ProMo tasks, mostly interactive editors.

\footnotetext{
${ }^{6}$ The combination "variables \& equations" is somewhat misleading. The term "equation" is commonly used to represent "variable" := "expression", where the "expression" is a function of variables and the ":=" stands for define. "Variables \&
} 
The expert section has two main tasks: (i) to generate the basic building blocks and link them to the graphical objects; (ii) to compile the behaviour equations to make them available for the task factory, and the OWL ontology as an extension to the EMMO [2].

ProMo's extension of the EMMO defines strictly a new variable as a function of existing variables. The Hamiltonian framework is the basis for mechanical systems and their configuration space, while contact geometry serves the same purpose for the thermodynamic systems. This approach allows us to generate all variables on a clean mathematical background. It has the advantage that at the basis is a minimal number of variables for which the units and dimensionalities are defined. The definition of new variables based on the existing variable allows for proper propagation of both the units and the dimensionalities [5]. The sequence for the definition of an equation is:

1. Choose level in the domain tree (Figure 2).

2. Define fundamental states, tokens in capacities, with indexes and physical units.

3. Define constants also with indexes and physical units.

4. Define networking-related matrices; the incidence matrix to capture the graphs and projections for mapping operations.

5. Select variable class and add variable and right-hand-side expression as a function of existing variables.

The result is a lower-triangular bipartite graph of variables and right-hand-side expressions.

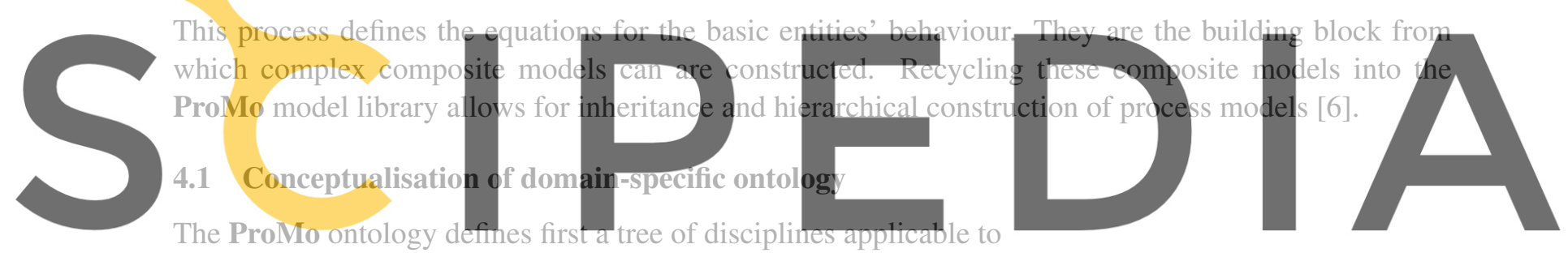

the modelling domain. Figure 2 shows the discipline tree for the ex-

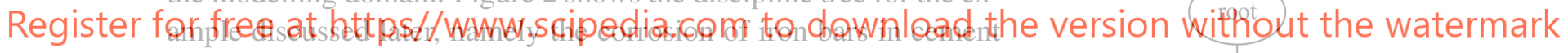

pillars. The model is one of mass transfer without any energy ef-

fects included, thus a very elementary model. We only need two phases, namely solid and liquid. Next, the ontology defines for each node elements for establishing the structure of the model and another branch defining the variable classes that apply for the branch defined by the node.

The equation editor loads the ontology providing the structure for the variable \& equation bipartite graph. The variable \& equations' definition starts with the base set of states, the base variables in physics' configuration spaces. Since these base sets are sufficient to define all variables via equations in the respective domain, namely

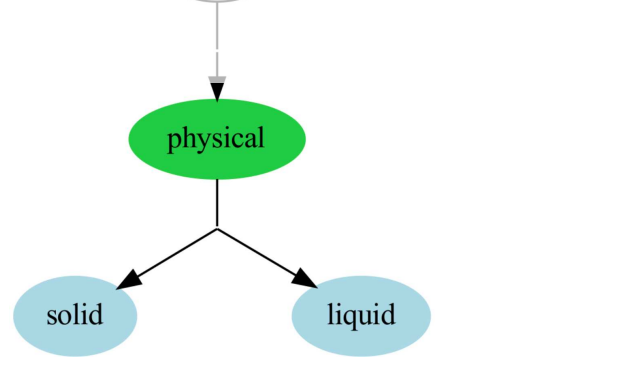

Figure 2: Domain tree corresponding to the simple corrosion simulation example. mechanical and thermodynamic domain, the result is a consistent set of variable \& equations. One only defines the units and dimensions of the base state set. The parser

expressions" would thus be a better terminology but is not what is commonly used. 


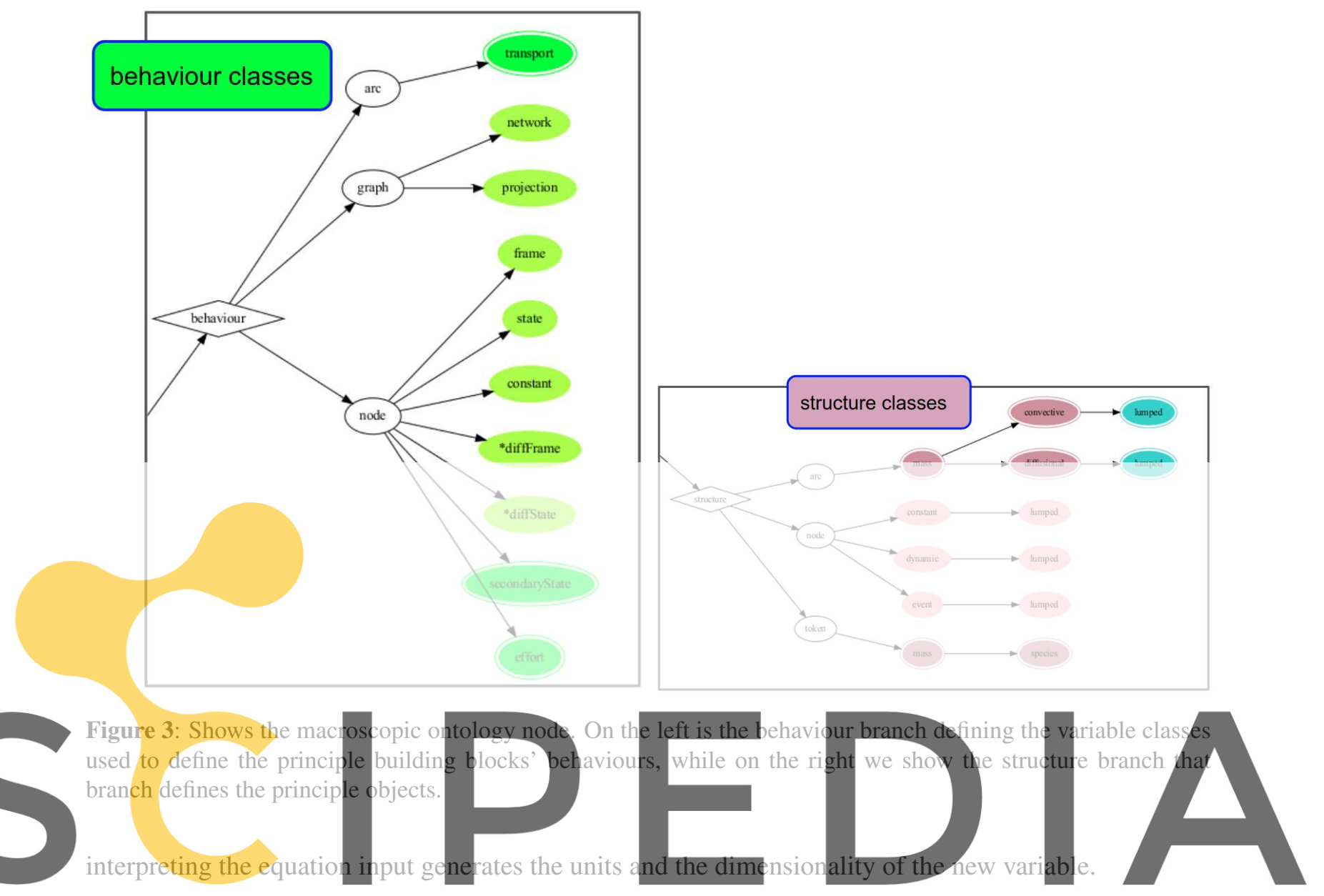

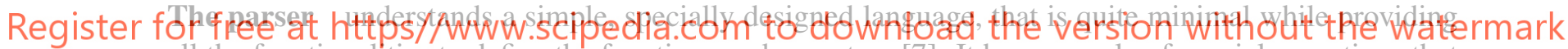
all the functionalities to define the functions and operators [7]. It has a couple of special operations that implement the index handling, which defines the dimensionality of the mathematical objects. ProMo uses block-objects, like vectors and matrices, to handle variable-length species sets or other token-related objects. The parser generates an abstract syntax tree for each of the right-hand-side expressions. A template machine also uses the abstract syntax tree to generate output code in different languages, like python, C++, MatLab, and LaTex, latter for documentation.

Reasoner The expert then attaches the state equations of the base entities to graphical objects. Once a state has been attached to a base entity, a reasoner analyses the bipartite tree starting with the state. It allows the definition of reduced bipartite graphs, to choose between alternative definitions for a variable or to truncate the graph. It also provides a list of the leave nodes which need to be initialised.

Those are then used in the graphics-based editor to define composite models. Defining the plant model is the task of the translator. Once established, it needs to be instantiated and coupled with the appropriate numerical solver. At this point, the translator passes the program on to the user, who then uses it to solve the defined problem. 


\subsection{Extending EMMO}

The parser/compiler also generates an OWL file. ProMo first imports EMMO from the public domain and append variables and equations in using the internal notation using the python module Olwready2. The thus generated Python file produces the OWL file when executed. In this way, the EMMO is extended with the variables and the relations between them, and one defines the terminology for the variables, their dimensionality, units and their association to a discipline. Once all is embedded in the OWL as an extension of the EMMO, one can utilise reasoners and automatically generate the API to connect different programs via the semantic services utilising REST. The extension of the EMMO using the equations generated by ProMo also puts the variable definitions in the EMMO on solid ground: ProMo derives all the equations from a base state set and some constants. The variable \& equation multi-bipartite graph is systematically constructed.

\section{Semantic interoperabilty}

Computer systems that supports semantic interoperability are able to discover and exchange information with a shared meaning between. The approach to semantic interoperability as described in [3] is to consider the entire technology stack. At the base of the stack is a set of abstractions (i.e. concepts, relations, rules and axioms etc.) in the form of ontologies. Here, the EMMO consist of a high-level and mid-level ontologies which are further derived into domain ontologies. Here we find the vocabulary and descriptions of the specific domain mapped to higher level concepts. The next layer in the technology stack are the web protocols that allows for information to be cross-referenced and shared. A structure of interlinked data relevant for a unambiguously knowledg tions sits on top of this

link between the softwa data model is supported base, represent linked datá
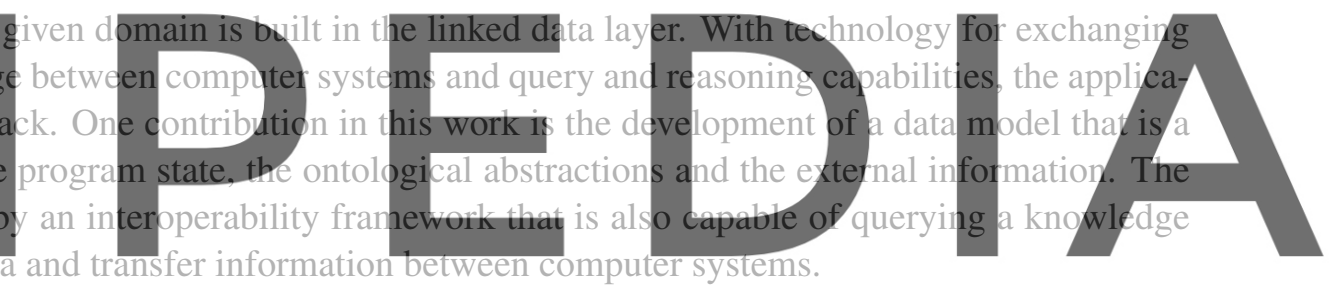

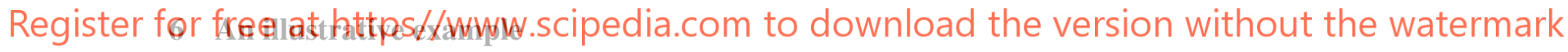

\subsection{Corrosion - an electrochemical process}

Corrosion is an extremely challenging issue that involves different fields of chemistry and physics. Especially corrosion of steel bars in concrete is a serious issue throughout the world [8]. This problem drains significant resources in both public and private sectors [9]. Generally, concrete provides a highly alkaline environment, which results in the formation of a passivating film that protects the steel from corrosion. However, the presents of chloride-ions can destroy the protection of the passive film. Especially this situation could be clear observed in the maritime environment, where chloride-ions diffuse into the concrete structures from the seawater. Moreover, chloride-ions may be intentionally added, most often as a constituent of accelerating admixtures. Dissolved chloride-ions also may penetrate unprotected concrete in structures exposed to deicing salts. If the chloride concentration exceeds a critical limit near the steel bar, the passive film breaks down, and the steel bars are no longer protected against corrosion.

Different factors influence the rate of corrosion of steel in concrete. In the first place are the environmental factors. Both oxygen and moisture must be present for electrochemical corrosion to occur [9]. Reinforced concrete with significant gradients in chloride-ion content is vulnerable to corrosion, espe- 
cially if subjected to wetting and drying cycles. Other factors that affected the rate and level of corrosion are heterogeneities in the concrete and the steel, $\mathrm{pH}$ of the concrete pore water, carbonation of the cement paste, cracks in the concrete, stray currents, and galvanic effects due to contact between dissimilar metals included in the construction. Thus, materials design features play an essential role in the corrosion of metals and their alloys. Mix proportions, depth of cover the metal, crack control measures, and implementation of measures designed specifically for corrosion protection and optimisation of corrosion protection are some of the factors that control the onset and rate of corrosion.

The most common form of iron corrosion in an aqueous medium is an electrochemical process [10], where an anode (where electrochemical oxidation takes place), a cathode (where electrochemical reduction occurs), an electrical conductor, and an aqueous medium must be present. Any metal surface on which corrosion occurs is a composite of anodes and cathodes electrically connected through the body of metal itself. Reactions at the anodes and cathodes are referred to as "half-cell reactions".

Let's consider a simplified description of a corrosion process, namely a steel bar in concrete in a maritime environment (in saltwater). The example demonstrates how we construct models in ProMo. We also show how we realise the data exchange in simulations of complex materials combinations.

Figure 4 shows the physical arrangement in a simplistic form. For this contribution we consider the simple corrosion process of iron in aggressive environment. At the anode, which is the negative pole, iron is oxidized to ferrous ions:

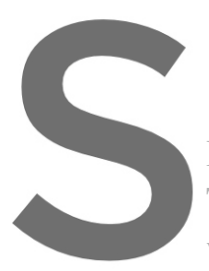

Herewith the Standard Redox P

The Standard Redox Potential when the metal is connected to a hydrogen electro
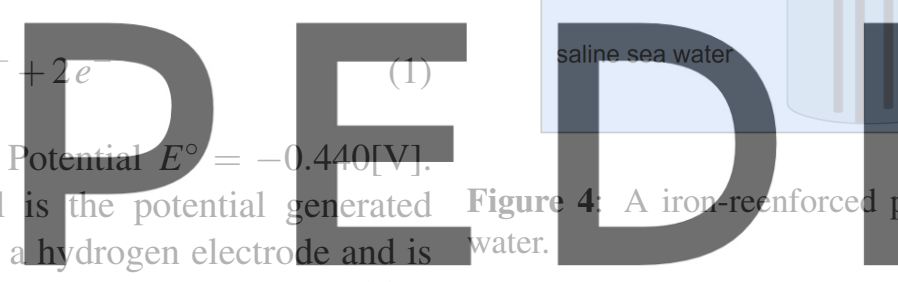
$A$ one method of expressing electromotive forces. The $\mathrm{Fe}^{++}$in Eq. 1 is subsequently changed to oxides of

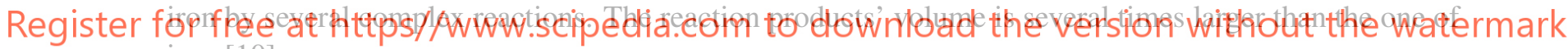
iron [10].

In an acid medium, the reaction taking place at the cathode and reduces hydrogen ions to hydrogen. However, concrete has a very high $\mathrm{pH}$ and usually has an adequate supply of oxygen, so the cathodic reaction is [9]:

$$
\frac{1}{2} \mathrm{H}_{2} \mathrm{O}+\frac{1}{4} \mathrm{O}_{2}+e^{-} \rightleftharpoons \mathrm{OH}^{-}
$$

Here the Standard Redox Potential $E^{\circ}=0.401[\mathrm{~V}]$.

The corroding iron part has an open circuit potential, also called a rest potential, related to the Standard Redox Potential of the reactions in Eq. 1 and 2, to the composition of the aqueous medium, the temperature, and the polarisation of these half-cells.

The following oxidation reaction also occurs in the presence of water:

$$
4 \mathrm{Fe}^{2+}+\mathrm{O}_{2} \rightarrow 4 \mathrm{Fe}^{3+}+2 \mathrm{O}^{2-}
$$


In addition, the multi-step reactions affect the corrosion product formation:

$$
\begin{aligned}
& \mathrm{Fe}^{2+}+2 \mathrm{H}_{2} \mathrm{O} \rightleftharpoons \mathrm{Fe}(\mathrm{OH})_{2}+2 \mathrm{H}^{+} \\
& \mathrm{Fe}^{3+}+3 \mathrm{H}_{2} \mathrm{O} \rightleftharpoons \mathrm{Fe}(\mathrm{OH})_{3}+3 \mathrm{H}^{+}
\end{aligned}
$$

as well as:

$$
\begin{aligned}
\mathrm{Fe}(\mathrm{OH})_{2} & \rightleftharpoons \mathrm{FeO}+\mathrm{H}_{2} \mathrm{O} \\
\mathrm{Fe}(\mathrm{OH})_{3} & \rightleftharpoons \mathrm{FeO}(\mathrm{OH})+\mathrm{H}_{2} \mathrm{O} \\
2 \mathrm{Fe}(\mathrm{OH}) & \rightleftharpoons \mathrm{Fe}_{2} \mathrm{O}_{3}+\mathrm{H}_{2} \mathrm{O}
\end{aligned}
$$

In this contribution, the simplification of the corrosion process is considered for model construction (see Figure 5).
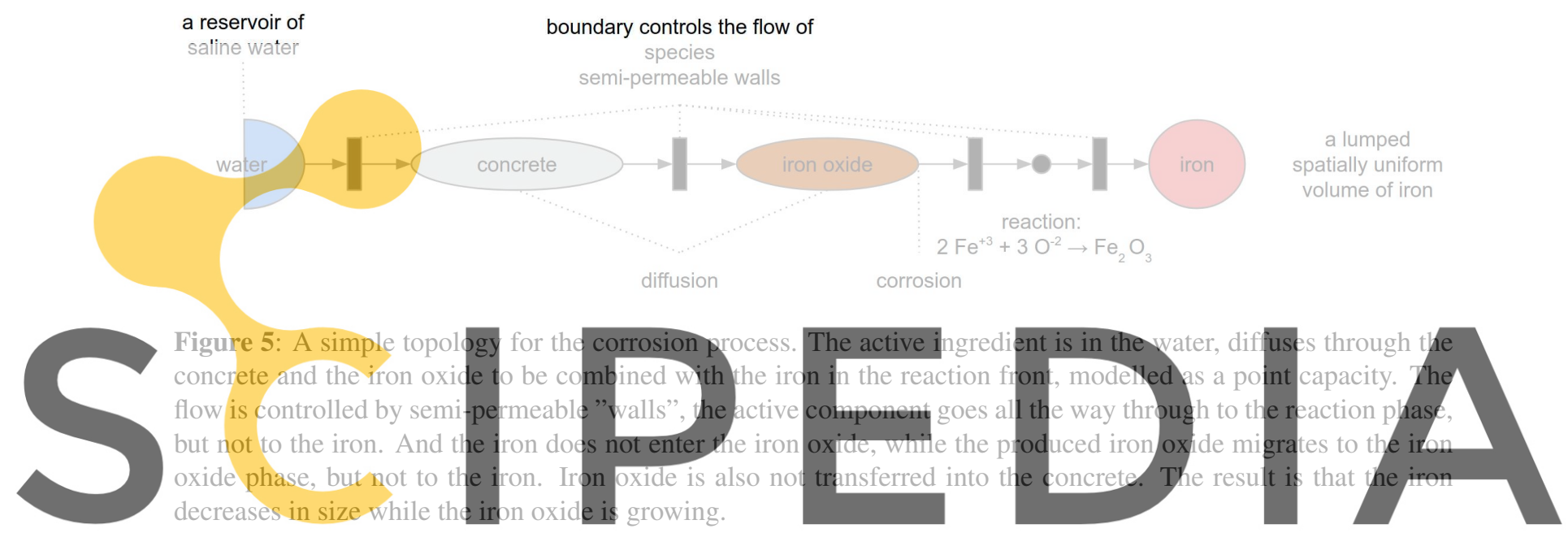

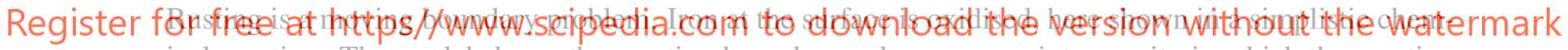
ical reaction. The model shows the reacting boundary volume as a point capacity in which the reaction takes place. The iron enters this point capacity from the right. It is taken from the iron, represented as a lumped capacity, and the rust is moving to the left adding to the rust domain. Consequently, the iron bar is shrinking, while the rust domain is growing.

As follows from the chemical equations, oxygen availability is one of the main controlling factors for steel corrosion. Moreover, the rate of oxygen diffusion through concrete is significantly affected by the cement's water contents. However, suppose the steel passivity is destroyed. In that case, conditions can lead to steel reinforcement's corrosion, notably, in those part of a concrete structure exposed to periods of intermittent wetting and drying. Moreover, if the concrete is continuously water-saturated, the rate of steel corrosion could be very slow, although chlorides are present in the environment. This phenomenon can be explained by the fact that dissolved oxygen will primarily diffuse in the water phase. In partly dry concrete, the diffusion of gaseous oxygen is much faster [9].

The effect of salt on the corrosion rate is significant. It is well known, that corrosion increased as the sodium chloride concentration increased until a maximum is reached [10]. Beyond this, the rate of corrosion decreased despite the increased chloride ion concentration. This change in the relationship between 
corrosion and sodium chloride concentration is attributed to the reduced solubility and diffusivity of oxygen and, therefore, oxygen availability to sustain the corrosion process.

\subsection{Salinity}

The quantity salinity is used as a measure of the salt level in seawater within oceanography. However, salinity can be measured in many different ways. It is only well-defined if its value is provided alongside a description of how it is measured. EMMO embraces this implicit requirement and defines a measurement as a semiosis process using the semiotic theory of Charles S. Peirce [11]. According to Peirce, is a semiosis process a triadic process or action involving three basic semiotic elements; an object, a sign representing the object and an interpretant, which is another sign that represents the interpreted sign of the same object. This is formalised in EMMO as a semiosis process having three participants, a sign (interpretant), an object and an interpreter that produces the interpreted sign.

Referring to Figure 6, lets take practical_salinity as an example. It is measured quantitative property determined through a practical_salinity_measurement of the electrical conductance of sea water, where the practical_salinity_measurement has the participants; practical_salinity (sign), sea_water (object) and conductivity meter (interpreter). Note that a semiosis processes can always be divided up into subprocesses, where the interpretant on the first process is the sign of the following process.
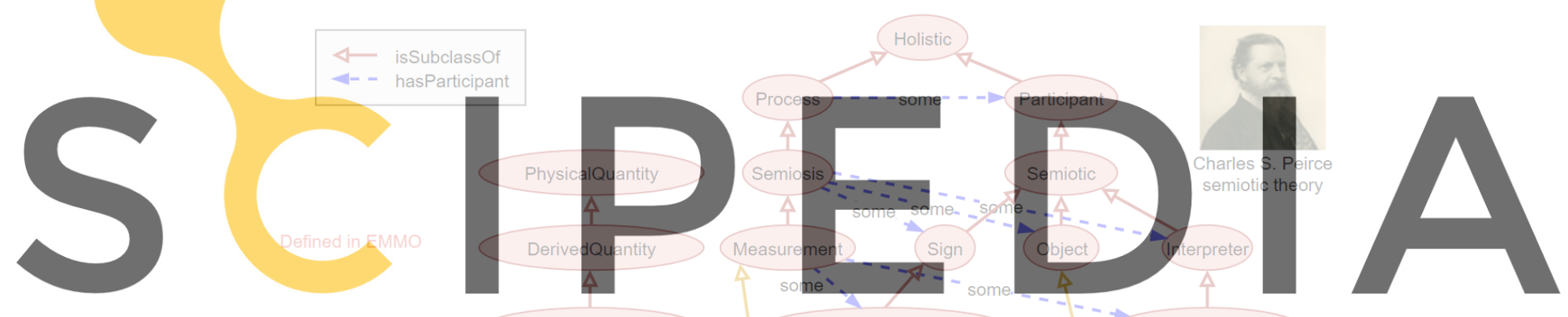

Register for free at https//www.scipedia.com to download the version without the watermark

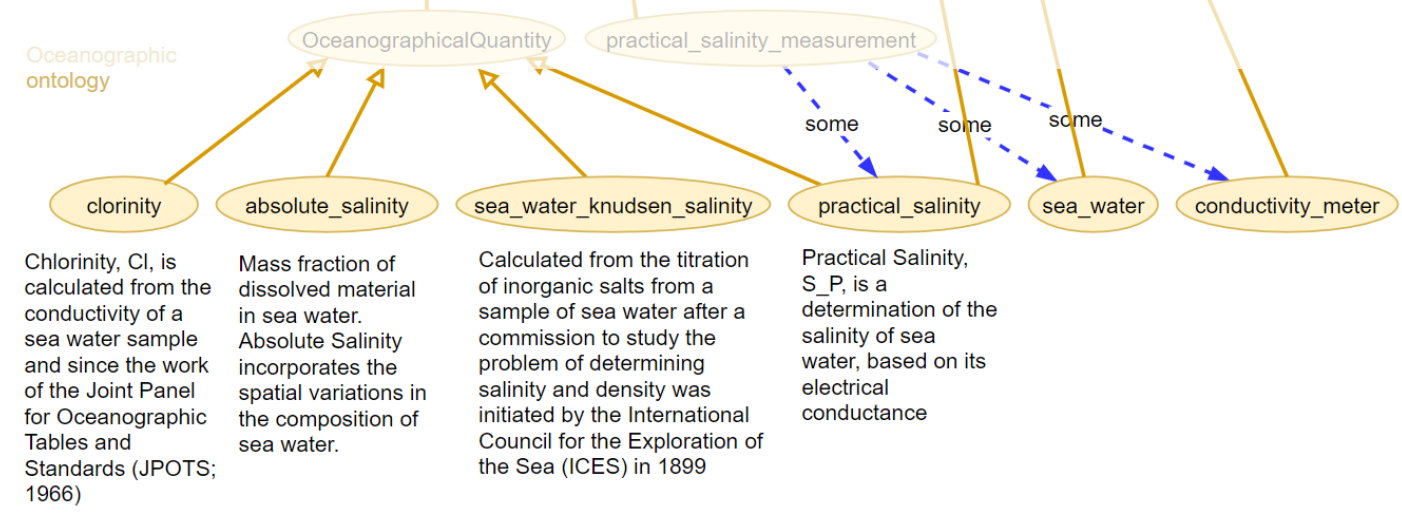

Figure 6: Ontological description of different ways to measure salinity, based on EMMO and Peirce semiotics. 
Making salinity available in simulations ProMo allows for the definition of new domains capturing discipline-specific knowledge. The logical approach to include such information as salinity would be to define an additional domain in the domain tree (Figure 2). One would define the new domain, call it "materialDataBase" in an inter-relation to the domain "physical". Within the domain "materialDataBase" one can then implement the appropriate reference to the respective information pair (measurement technology, value) and provide transformations if necessary.

\subsection{Data exchange approach}

There are different possible approaches to data exchange. In the NanoSim project (A Multi-scale Simulation-Based Design Platform for Cost-Effective CO2 Capture Processes using Nano-Structured Materials funded by the European Union's FP7-NMP research and innovation programme under grant agreement no 604656), an early implementation of the interoperability framework SOFT demonstrated a data-centric approach for exchanging information between simulators to solve multi-scale/multi-physics problems. The SOFT framework was responsible for connecting and exchange information between heterogenous datasources. Only a reference to a collection of entities that was produced by one simulation step was passed to the next simulation step. At this stage, the simulation software would instantiate its own entities and include them in the collection and fetch the information from the data source(s) via the SOFT framework. In the case data models that represented output from one simulations step didn't match the data models representing the input in the next simulation step, a transformation had to be inserted in between. This transformation is in principle no different from any other computational steps that transforms a set of inputs into a set of outputs. By this logic it is possible to infer how compatible two simulators are, by examining their data-models. In our updated model we can take this one step further by mapping the data-models to the ontological concepts that defines the state of the physical quantities of the composite models defined by tools such as ProMo. This mapping allows data to be serialized from heterogeneous data sources [3] into the process simulator ensuring the information is interpreted correctly .

Another approach is to pass information between the different simulation tools. This information will need to contain data and metadata that either maps to an external ontological representation, or include the ontology in the data payload.

\section{Discussion \& conclusions}

We have shown that ontologies play an essential role in structuring discipline-specific knowledge. Ontologies provide a classification of the involved items and a taxonometry, fix the terminology for the application, thereby improving communication. Ontologies describe the relations that exist between the items and serve the purpose of a "building instruction". Latter is utilised when constructing the variable \& equation set that provides a behaviour description of the basic building blocks for each involved discipline. Furthermore, it is also used when creating models using these building blocks. We utilise the ontology, expanded by the building blocks' behaviour description, to automatically generate the involved programs' APIs. The generation of the executable code realising the simulation becomes largely automatic and thus reduces the programming effort significantly.

The common use of ontologies in data mining and pattern search is rather unbalanced with the use of ontologies as building instructions. We are the opinion that the power of the latter is largely undervalued. 
While the EMMO provides the terminology and classification for materials, ProMo adds the relations for the behaviour description of the basic physical entities and beyond the ones utilised in simulations, namely control and analytical tools. The automation of code generation will open the field of computing complex multi-disciplinary and multi-scale processes to a much larger public while reducing the time from model to simulation by two orders of magnitude.

\section{Acknowledgments}

The authors acknowledge the following European Union's research and innovation projects for the financial support: (i) OntoTRANS, H2020-DT-NMBP-10-2019, Grant agreement 862136; (ii) MarketPlace, H2020-NMBP-252017, Grant agreement 760173; (iii) FORCE, H2020-NMBP-23-2016, Grant agreement 721027; (iv) VIPCOAT, H2020-DT-NMBP-11-2020, Grant agreement 952903.

\section{REFERENCES}

[1] Dykeman, D., Hashibon, A., Klein, P., and Belouettar, S. (2020, September 22). Guideline Business Decision Support Systems (BDSS) for Materials Modelling. Zenodo: https://doi.org/10.5281/zenodo.405400

[2] Ghedini E., Goldbeck G., Friis J., Hashibon A. and Schmitz G.J. European Materials \& Modelling Ontology, https://github.com/emmo-repo/EMMO.

[3] Hagelien, Thomas F and Preisig, Heinz A and Friis, Jesper and Klein, Peter and Konchakova, Natalia A practical approach to ontology-base data modelling for semantic interoperability 14th World Congress on Computational Mechanics, ECCOMAS Congress 2020, 11-15 January 2021 F. Chinesta, R. Abgrall, O. Allix and M. Kaliske (Eds)

[4] MoDeNa MODENA FP7-NMP-Specific Programme "cooperation": Nanosciences, Nanotechnologies, materials and new Product Technologies Grant agreement ID: 604271

[5] Preisig, H.A. Constructing and maintaining proper process models. Comp \& Chem Eng 2010, 34(9), 1543-1555. doi:https://doi.org/10.1016/j.compchemeng.2010.02.023.

[6] Preisig, H A, Ontology-based process modelling - with examples of physical topologies, MDPI, Processes, submitted (2021).

[7] Elve, A.T.; Preisig, H.A. From ontology to executable program code. Comp \& Chem Eng 2018. doi:https://doi.org/https://doi.org/10.1016/j.compchemeng.2018.09.004.

[8] Mohammed, T.U., Otsuki, N., and Hamada, H. Corrosion of Steel Bars in Cracked Concreteunder Marine Environment Journal of Materials in Civil Engineering. (2003) 15, https://doi.org/10.1061/(ASCE)0899-1561(2003)15:5(460)

[9] American Concrete Institute (ACI) Corrosion of Metals in Concrete Reported by Committee 222, ACI 222-96, Detroit, https://de.slideshare.net/MOHAMMEDSABBAR2/222-r-96-corrosionof-metals-in-concrete

[10] Revie, R. W., Uhlig, H. H. Corrosion and Corrosion Control: An Introduction to Corrosion Science and Engineering, Fourth Edition, John Wiley \& Sons (2008)

[11] Peirce, C.S. Studies in Logic, by Members of The Johns Hopkins University (1883) Ed. Charles S. Peirce. Boston: Little Brown. 